\title{
Distinctive expression pattern of interleukin-17 cytokine family members in colorectal cancer
}

\section{Al-Samadi, Ahmed}

2016-02

Al-Samadi , A, Moossavi , S , Salem , A , Sotoudeh , M , Tuovinen , S M , Konttinen , Y T , Salo , T \& Bishehsari , F 2016 , ' Distinctive expression pattern of interleukin-17 cytokine family members in colorectal cancer ' , Tumor Biology , vol. 37 , no. 2 , pp. 1609-1615 . https://doi.org/10.1007/s1327

http://hdl.handle.net/10138/224025

https://doi.org/10.1007/s13277-015-3941-x

publishedVersion

Downloaded from Helda, University of Helsinki institutional repository.

This is an electronic reprint of the original article.

This reprint may differ from the original in pagination and typographic detail.

Please cite the original version. 


\title{
Distinctive expression pattern of interleukin-17 cytokine family members in colorectal cancer
}

\author{
Ahmed Al-Samadi ${ }^{1,2} \cdot$ Shirin Moossavi $^{3}$ • Abdelhakim Salem ${ }^{1} \cdot$ Masoud Sotoudeh $^{4}$. \\ Sarianna M. Tuovinen ${ }^{1} \cdot$ Yrjö T. Konttinen ${ }^{1,5,6} \cdot$ Tuula Salo $^{2} \cdot$ Faraz Bishehsari $^{7}$
}

Received: 6 July 2015 / Accepted: 17 August 2015 / Published online: 25 August 2015

(C) International Society of Oncology and BioMarkers (ISOBM) 2015

\begin{abstract}
Colorectal cancer (CRC) is one of the most common cancers in both genders. Even though interleukin (IL)17A was shown to play an important role in intestinal tumourigenesis and CRC, other IL-17 family members were not studied well. We therefore studied the expression of IL-17 cytokine family members in CRC. Ten healthy colons and ten CRC mucosa were immunostained for IL-17B, IL-17C, IL17E, and IL-17F, and their receptors IL-17RA, IL-17RB, and IL-17RC. Double immunofluorescence staining of the CRC mucosa was done for IL-17B with markers of neutrophils, endothelial cells, macrophages, $\mathrm{T}$ cells, mast cells, or fibroblasts. While IL-17B was increased in CRC with a strong presence both in the epithelial and stromal compartments, IL-17C showed different expression depending on the grade of differentiation and IL-17E remained unchanged. In contrast, IL-17F was decreased in CRC compared to healthy control. Colon epithelial cells stained positive for IL-17RA, IL17RB, and IL-17RC in both healthy control and CRC. Neutrophils were the main source of IL-17B in the stroma. IL-17
\end{abstract}

Yrjö T. Konttinen passed away during the preparation of this study.

Electronic supplementary material The online version of this article (doi:10.1007/s13277-015-3941-x) contains supplementary material, which is available to authorized users.

Shirin Moossavi

shirin.moossavi@gmail.com

1 Department of Medicine, Institute of Clinical Medicine, University of Helsinki, Helsinki, Finland

2 Department of Oral and Maxillofacial Diseases, University of Helsinki, Helsinki, Finland

3 Digestive Oncology Research Center; Digestive Disease Research Institute, Tehran University of Medical Sciences, Shariati Hospital, North Amirabad Ave., Tehran 14117, Iran family members demonstrated distinct expression patterns in $\mathrm{CRC}$, suggesting a differential role exerted by each member in colon carcinogenesis.

Keywords IL-17 · Colorectal cancer $\cdot$ IL-17B $\cdot$ Neutrophil

\section{Introduction}

Colorectal cancer is (CRC) is the second most common cancer in women and third most common cancer in men worldwide [1]. Despite the significant improvement in our understanding of the genetic alterations in the course of colorectal adenoma-carcinoma progression, the pathogenesis of tumour initiation/promotion is not fully understood. Chronic inflammation is a major driving force in the development and progression of colitis-associated cancer (CAC) [2]. Th17 and IL-17 cytokine family are emerging as prominent players in gastrointestinal mucosal

4 Pathology Department, Tehran University of Medical Sciences, Tehran, Iran

5 Department of Medicine, Helsinki University Central Hospital, Helsinki, Finland

6 ORTON Orthopedic Hospital of the Invalid Foundation, Helsinki, Finland

7 Division of Digestive Diseases, Rush University Medical Center, Chicago, IL, USA 
immunity in health and disease [3,4]. Specifically, their role in CRC is under investigation [5-7].

IL-17 family of cytokines are comprised of IL-17A-F, which bind to IL-17 receptors (IL-17R) A-E [3]. The role of IL-17A has been intensively studied in intestinal tumourigenesis and CRC [8-13]. Adenomatous polyposis coli $(A p c)^{\mathrm{min} /+} \mathrm{IL}-17 \mathrm{~A}^{-/-}$mice were shown to have a reduced number of tumours in the small intestine and colon compared to $A p c^{\mathrm{min} /+}$ control [14]. Also, the expression of IL-17A is increased during spontaneous colorectal tumourigenesis in CDX2promoter regulated Cre (CPC)-APC mouse model [10]. Treatment of enterotoxigenic Bacteroides fragilis (ETBF)-colonised $A p c^{\mathrm{min} /+}$ mice with anti-IL-17A antibody results in reduced gastrointestinal intraepithelial neoplasia (GIN) foci and tumour formation [15]. Other reports have also shown similar effect for IL-17A [16, 17]. Overall, IL-17A seems to have a pro-tumourigenic effect.

Despite the wealth of information on IL-17A in colitis and CRC, very little is known about other IL-17 family members in general and in CRC in particular. Therefore, our main objective was to assess tissue expression of IL-17B, IL-17C, IL-17E, and IL-17F and their receptors in sporadic CRC and to identify their cellular source in CRC microenvironment.

\section{Materials and methods}

\section{Patients and specimens}

Ten formalin-fixed, paraffin-embedded CRC tissues were selected from patients who underwent surgical resection from February 1998 to September 2003 in two major hospitals in Tehran (Atieh and Mehr). Patients had no history of inflammatory bowel disease, familial adenomatous polyposis, or hereditary non-polyposis CRC (HNPCC) and had not undergone preoperative chemotherapy or radiotherapy. The pathologic diagnosis of colorectal adenocarcinoma had been independently confirmed by the Pathology Unit, Shariati Hospital, Tehran University of Medical Sciences, Tehran, Iran, and the Pathology Section of the Center of Excellence on Ageing (CeSI), Chieti, Italy [18]. Patients' characteristics are summarised in Table S1. Healthy controls were selected from individuals who underwent elective screening colonoscopy at Masoud Clinic, Tehran, Iran, and had no visible lesion in the colonoscopy. This study was approved by the Ethics Committee of the Digestive Disease Research Institute, Tehran University of Medical Sciences. Written informed consent had been obtained.

\section{Immunostaining}

Immunohistochemistry and double immunofluorescence staining were conducted as previously described [19]. The following antibodies were used for immunostaining: $1 \mu \mathrm{g} / \mathrm{ml}$ goat anti-human IL-17B IgG (R\&D System, Minneapolis, MN, USA), $1 \mu \mathrm{g} / \mathrm{ml}$ goat anti-human IL-17C IgG (R\&D System), $1 \mu \mathrm{g} / \mathrm{ml}$ rabbit anti-human IL-17E IgG (Bioss, Woburn, MA, USA), $1 \mu \mathrm{g} / \mathrm{ml}$ goat anti-human IL-17F IgG (R\&D System), $1 \mu \mathrm{g} / \mathrm{ml}$ goat anti-human IL-17RA IgG (R\&D System), $1 \mu \mathrm{g} / \mathrm{ml}$ goat anti-human IL-17RB IgG (R\&D System), or $1 \mu \mathrm{g} / \mathrm{ml}$ goat anti-human IL-17RC IgG (R\&D System). Slides were washed in PBS for three times for 5 min each and then incubated in biotin-conjugated rabbit anti-goat IgG secondary antibody. Slides were washed again three times for 5 min each and incubated in avidin-biotin-peroxidase complex (Vectastain Elite ABC kit; Vector Laboratories, Burlingame, CA, USA) for $1 \mathrm{~h}$ at room temperature. Colour was developed using $0.006 \% \mathrm{H}_{2} \mathrm{O}_{2}$ substrate and $0.023 \%$ 3,30-diaminobenzidine tetrahydrochloride (DAB) chromogen for $10 \mathrm{~min}$ at room temperature. For negative staining control nonimmune goat and rabbit IgG was used at the same concentration and instead of the primary antigen-specific antibodies.

\section{Double immunofluorescence staining}

Slides were deparaffinised and antigen retrieval was done as in the immunostaining. Slides were treated with $0.5 \%$ Triton $\mathrm{X}-100$ for $10 \mathrm{~min}$ at room temperature and incubated in $10 \%$ monkey normal serum. Subsequently, slides were incubated in $5 \mu \mathrm{g} / \mathrm{ml}$ goat anti-human IL-17B IgG, $0.35 \mathrm{mg} / \mathrm{ml}$ rabbit anti-human neutrophil elastase (NE) IgG (Abcam, Cambridge, UK), $5 \mu \mathrm{g} / \mathrm{ml}$ monoclonal mouse anti-human CD3 IgG1 (Dako, Glostrup, Denmark), $0.5 \mu \mathrm{g} / \mathrm{ml}$ mouse anti human CD68 IgG1 (DAKO), $0.1 \mu \mathrm{g} / \mathrm{ml}$ mouse anti-human mast cell tryptase (MCT) IgG1 (MST, AbDSerotec, Oxford, UK), $22.5 \mu \mathrm{g} / \mathrm{ml}$ mouse anti-human CD31 IgG1 (DAKO), or $10 \mu \mathrm{g} / \mathrm{ml}$ mouse anti-human heat shock protein (HSP) 47 IgG2b (StressGen Biotechnologies Corp., Victoria, BC, Canada). Slides were washed in PBS three times for 5 min each and incubated in fluorescein-conjugated monkey anti-rabbit, fluorescein-conjugated monkey anti-mouse, and fluoresceinconjugated monkey anti-goat IgG secondary antibodies for $1 \mathrm{~h}$ at room temperature. Slides were washed again with PBS three times for $5 \mathrm{~min}$ each and nuclei were counterstained with 4',6-diamidino-2-phenylindole (DAPI) for $10 \mathrm{~min}$ at room temperature. Finally, slides were mounted in Vectashield ${ }^{\circledR}$ mounting medium (Vector Laboratories, Burlingame, CA, USA). For negative staining control non-immune goat $\operatorname{IgG}$, rabbit IgG, mouse IgG1, and mouse IgG2b was used at the same concentration and instead of the primary antigenspecific antibodies. 


\section{Microscopy and grading}

Stained tissue was analysed and photographed using Leica DM6000 B/M light microscope connected to a digital camera (DFC420 and DFC365FX; Leica Microsystems, Wetzlar, Germany). The number of IL-17B ${ }^{+}$cells in the stromal compartment was counted in three high-power fields and the results were reported as number of cells per square millimeter. Since, epithelium was found to be positive for all cytokines tested, we assigned staining intensity to the whole section rather than counting the number of positive cells, as a quantifiable measure. As image-processing software cannot distinguish between epithelial and stromal compartments in the colon tissue, we opted for visual scoring of the staining intensity of the epithelium. Immunostaining intensity of the epithelial compartment was graded by visual scoring as 0 (no staining),
1 (week staining), 2 (moderate staining), and 3 (strong staining).

\section{Statistical analysis}

Mann-Whitney $U$ test was used to assess the statistical significance. A $P$ value of less than 0.05 was considered significant.

\section{Results}

\section{Differential expression of IL-17 family cytokines in CRC}

There is no prior knowledge on the expression of IL-17 cytokine family members except IL-17A in CRC. Therefore, as a preliminary analysis, we investigated expression of IL-17B,

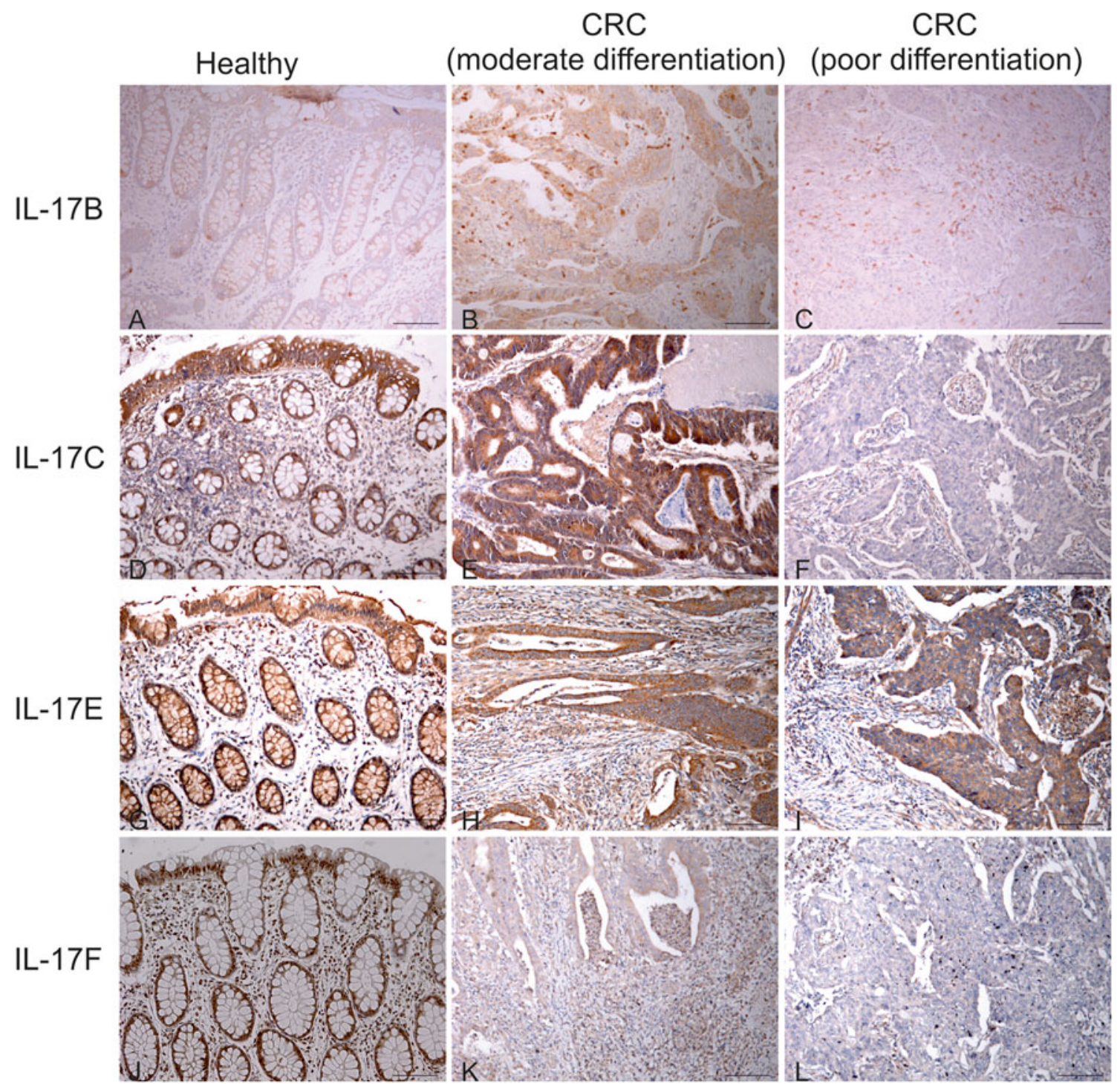

Fig. 1 IL-17 family cytokines expression in healthy colon and colorectal cancer (CRC). Healthy colon and CRC (moderate and poor differentiation) samples were immunostained for IL-17B (a-c), IL-17C (d-f), IL-17E (g-i), and IL-17F (j-l). Scale bar $=100 \mu \mathrm{m}$ 
$\mathrm{C}$, E, and $\mathrm{F}$ in ten CRC cases. IL-17B, IL-17C, IL-17E, and IL-17F had a cytoplasmic pattern of expression in normal colon and CRC. They are all expressed both by the epithelium and stromal components. We observed different expression levels of these cytokines in our samples.

We detected a weak expression of IL-17B in the epithelium and strong expression in some connective tissue cells in healthy colon (Fig 1a). IL-17B tissue expression was increased in CRC tumour cells with moderate and poor differentiation (Fig 1b, c). The number of IL-17B ${ }^{+}$stromal cells in CRC was higher than healthy control (Fig. S1).

IL-17C is expressed in colon epithelium [20]. We also observed that healthy colon epithelium stain positive for IL-17C. It seems that the surface epithelium is more strongly positive for IL-17C compared to deeply located epithelium. However, this observation might be due to lower volume of cytosol in goblet cells, which are more abundant in the crypts (Fig 1d). We observed a distinct pattern of IL-17C expression in CRC. Normal colon epithelium has a strong expression of IL-17C (Fig. 1d). While IL-17C was abundantly present in moderately differentiated CRC (Fig. 1e), the poorly differentiated cases were either faintly positive or negative (Fig 1f).

IL-17E (IL-25), another member of the IL-17 family, is more commonly studied in relation to allergic reactions and asthma. IL-17E is expressed in normal intestinal tract including the colon [21]. Our results showed a strong expression of IL-17E in both healthy and CRC epithelium and connective tissue cells with no difference between them (Fig 1gi).

IL-17F is the second most commonly studied member of the IL-17 family cytokines after IL-17A. IL-17F is present in healthy epithelium and some connective tissue cells (Fig 1j), in contrast to that epithelium staining is clearly weaker in moderate and poor grade CRC compared to healthy epithelium (Fig 1k, 1). IL-17F had a significantly lower staining intensity in CRC compared to that in the control (Fig. S2).

\section{IL-17 receptors expression in CRC}

We assessed the expression pattern of IL-17RA, IL-17RB, and IL-17RC in CRC compared to that in the control. We observed an epithelial expression of the three receptors in both CRC and healthy controls with no clear difference between them (Fig. 2).

\section{IL-17B is produced by neutrophils}

We had previously identified neutrophils to be positive for IL17B in synovial tissue [22]. In light of the strong stromal presence of IL17B in CRC samples, we used double immunofluorescence staining to identify cells positive for IL-17B in
Fig. 2 IL-17 cytokines receptors expression in healthy colon and colorectal cancer (CRC). Healthy colon and CRC samples were immunostained for IL-17RA (ab), IL-17RB (c-d), and IL-17RC (e-f). Scale bar $=100 \mu \mathrm{m}$

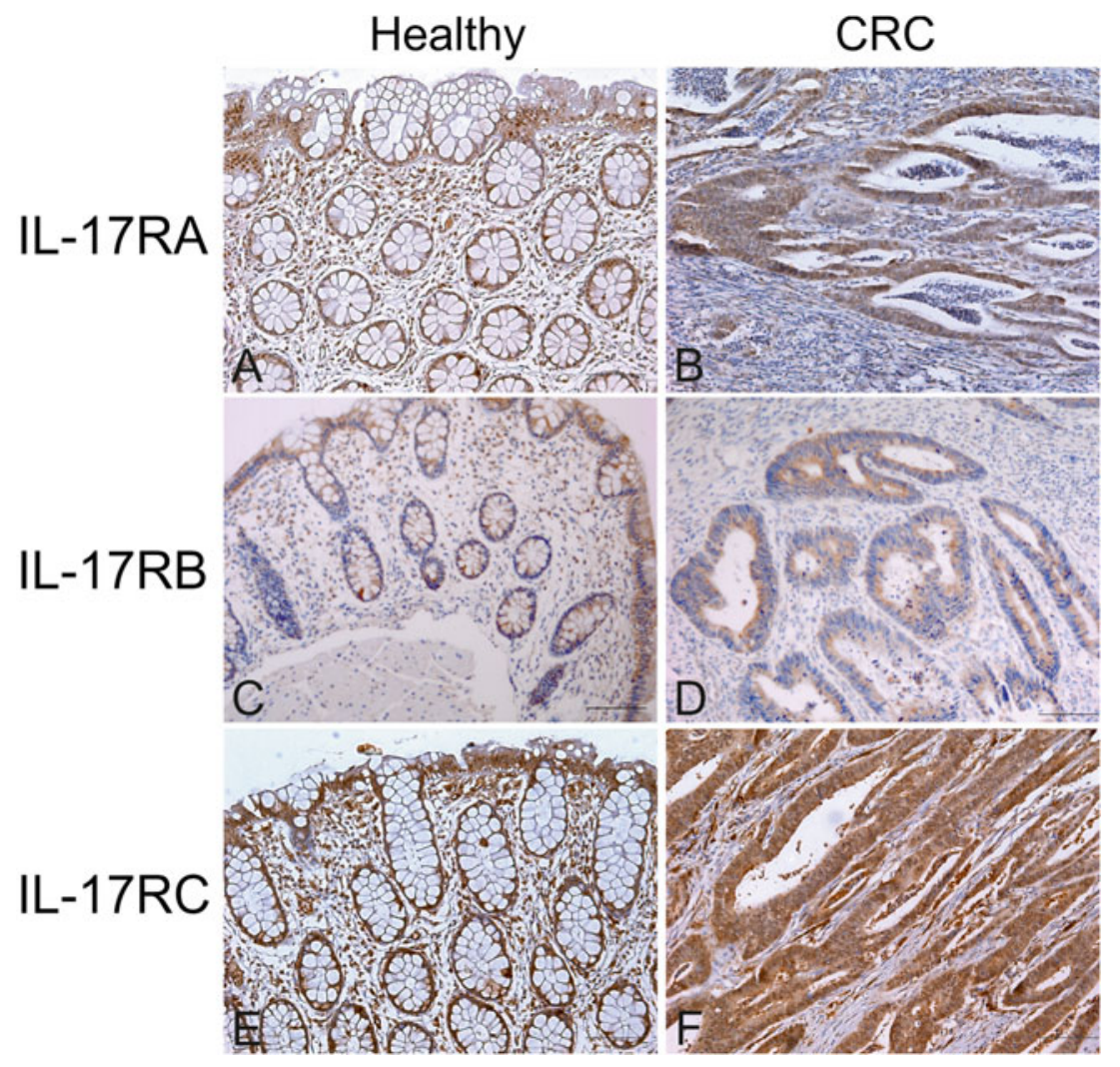


the connective tissue. Consistent with prior reports, we found that neutrophils are strongly present in tumour tissue while absent in normal tissue (data not shown). We also found that some neutrophils are positive for IL-17B (Fig. 3a). Although we observed that IL-17B ${ }^{+}$cells colocalised with the microvasculature, IL-17B did not colocalise with any other component of the stroma such as endothelial cells, myofibroblasts, macrophages, T cells, and mast cells (Fig. 3). It was previously shown that activated $\mathrm{CD}^{+} \mathrm{T}$ cells expressed IL-17 but not IL-17B, indicating a different source of IL-17B [23]. Our results suggest that IL$17 \mathrm{~B}$ is associated with neutrophils in CRC.

\section{Association of IL-17 with molecular features of CRC}

We also compared the epithelial intensity of IL-17B, C, E, and $\mathrm{F}$ in $\mathrm{CRC}$ cases according to gender, anatomic location of the tumour, microsatellite instability, and Duke staging. We did not detect a significant association between tissue expression of these cytokines and clinical features of CRC. However, potentially interesting differences were observed. For example, we observed a higher IL-17E intensity in left-sided tumours compared to right-sided tumours (Fig. S3).

\section{Discussion}

IL-17 family is consisted of six members, IL-17A-F. IL-17A has been studied in CRC and murine models of intestinal tumourigenesis [10, 12-14]. However, very little is known about other IL-17 cytokines expression in CRC. We investigated tissue expression of these cytokines and their receptors in sporadic CRC vs. healthy control. While IL-17B was increased in CRC, IL-17C showed different expression
Fig. 3 Double

immunofluorescence staining of IL-17B together with neutrophil marker (human neutrophil elastase, NE), endothelial cell marker (CD31), macrophage markers (CD68), T cells marker (CD3), mast cells marker (mast cell tryptase, MCT), or fibroblasts marker (heat shock protein 47 , HSP47). Colorectal cancer tissue was immunostained for IL-17B together with human NE (a), CD31 (b), CD68 (c), CD3 (d), MCT (e), and HSP47 (f). Nuclei were counterstained with DAPI. Scale bar $=50 \mu \mathrm{m}$
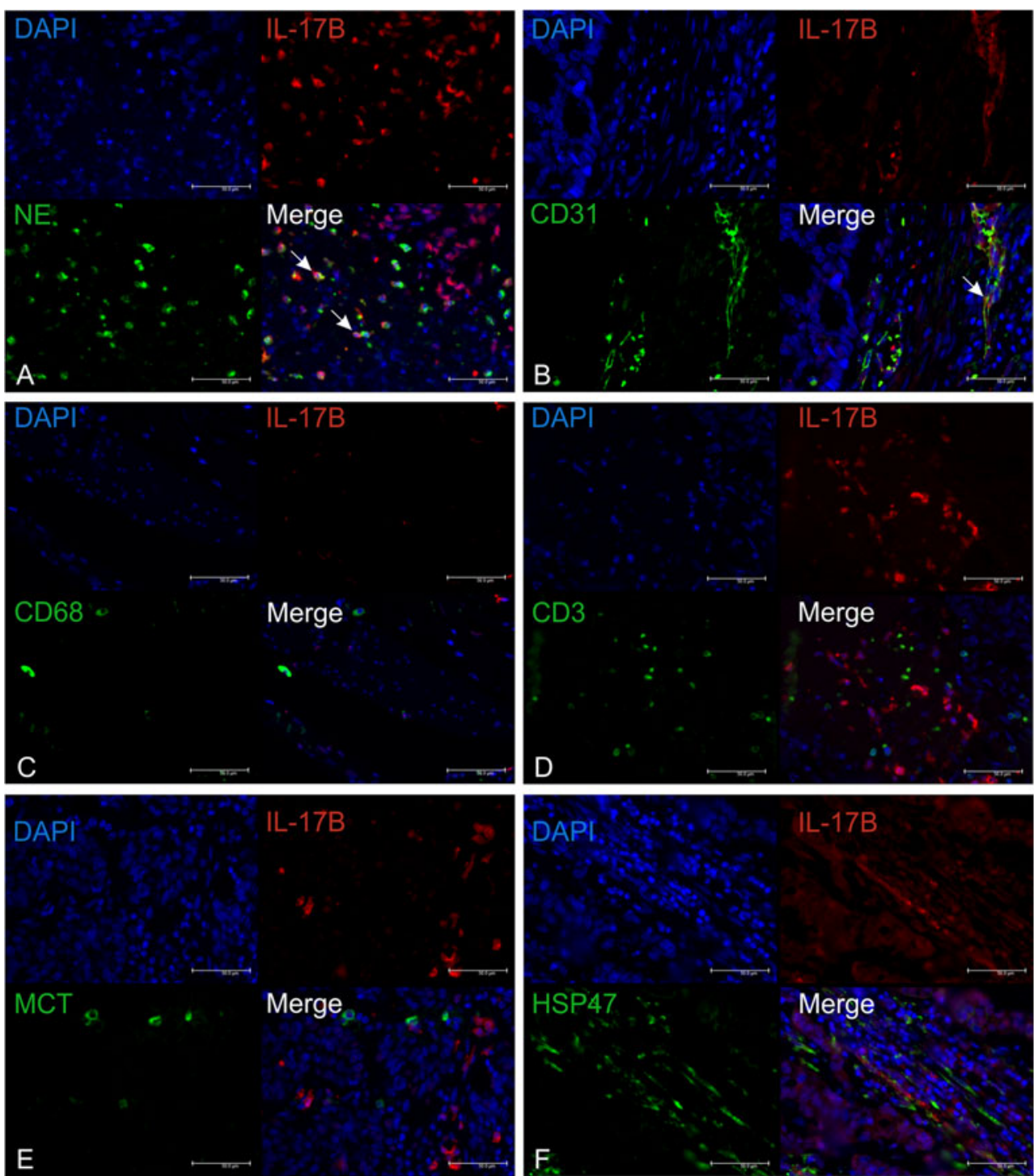
depending on the grade of differentiation and IL-17E remained unchanged. In contrast, IL-17F was decreased in CRC compared to healthy control. IL-17RA, IL-17RB, and IL-17RC are all expressed in both healthy control and CRC.

IL-17B was first cloned and molecularly characterised in 2000 [23]. In our work, IL-17B was increased in CRC mainly in the stromal compartment. Increased IL-17B in CRC can result in increased inflammation associated with cancer as IL-17B has been shown to induce IL-6 and IL-8 expression from colon subepithelial myofibroblasts [24]. IL-17RB epithelial expression in our study was not different between healthy control and CRC. An increased level of IL-17B, in light of an unchanged IL-17RB expression could result in an increase in the IL-17B-intitiated signaling in cancer tissue, which could potentially increase the tumourigenesis capacity as it was previously shown in breast cancer [25]. Consistent with an increased IL-17B in the stromal compartment, we found neutrophils as a source of IL-17B in CRC. We have previously identified neutrophils as the source of IL-17B in rheumatoid synovial tissue [22]. The functional significance of this observation needs to be further explored; however, it is possible that it may help in the recruitment and protumourigenic transformation of neutrophils in the tumour microenvironment [26, 27].

IL-17C has been reported to be increased at mRNA and protein level in CRC and mouse models of chemical-induced colitis-associated cancer (CAC) and $\mathrm{ApC}^{\mathrm{min} /+}$ model of spontaneous intestinal tumourigenesis [28]. We have found a comparable expression level of IL-17C in CRC and healthy controls; however, IL-17C expression tended to decrease in advanced cancer. This pattern suggests the implication of IL$17 \mathrm{C}$ in cancer progression rather than in carcinogenesis process.

IL-17E (IL-25) has been studied in colitis and IBD but not in CRC. IL-17E expression is significantly reduced in IBD [29]. IL-17E could prevent chemical-induced colitis if administered prior to induction or ameliorate the disease severity if administered after the induction [29-33]. Our results, however, showed a strong expression of IL-17E in both healthy control and CRC with no difference between them. Similarly, IL-17RA and IL-17RB, which form the heterodimer receptor complex for IL-17E, are abundantly expressed in the epithelium of CRC as well as normal tissue. To the best of our knowledge, this is the first report of the expression of IL17E in CRC; the functional significance of which needs to be explored.

IL-17F is the second most commonly studied member of the IL-17 family cytokines after IL-17A. IL-17F has a protective role against intestinal and liver tumourigenesis [34, 35]. We observed a decrease in the expression of IL-17F in CRC in parallel with the reduction in the degree of differentiation, which is in accordance with Tong et al. who also showed a decrease in the expression of IL-17F in CRC [34].
In conclusion, for the first time, we studied the expression of four members of the IL-17 family and their respective receptors in sporadic CRC and healthy controls. In this preliminary study, we observed differential expressions of IL-17 family members in CRC, while their receptors are abundantly expressed in normal and cancer tissue. The differential expressions of these cytokines require to be corroborated in larger sample size. Nevertheless, the differential expressions of these cytokines in CRC may represent a mechanism by which the tumourigenesis capacity is regulated. Indeed, there seems to be differences in the expression level of these cytokines based on clinical features of the disease. Different roles of IL-17B, $\mathrm{C}$, and $\mathrm{F}$ in the onset and severity of collagen-induced arthritis have been demonstrated [36]. Although, the collective effect of these cytokines on intestinal tumourigenesis is not known; it is conceivable that they play distinct roles in fine-tuning the tumour initiation and/or promotion at different stages of CRC.

Acknowledgments This work was supported by the Finska Läkaresällskapet, the Paulo Foundation, the Sigrid Jusélius Foundation, the Finnish Dental Society Apollonia, and the Digestive Disease Research Institute.

Conflicts of interest None

\section{References}

1. Jemal A, Bray F, Center MM, Ferlay J, Ward E, Forman D. Global cancer statistics. CA Cancer J Clin. 2011;61(2):69-90.

2. Bernstein CN, Blanchard JF, Kliewer E, Wajda A. Cancer risk in patients with inflammatory bowel disease: a population-based study. Cancer. 2001;91(4):854-62.

3. Pappu R, Ramirez-Carrozzi V, Sambandam A. The interleukin-17 cytokine family: critical players in host defence and inflammatory diseases. Immunology. 2011;134(1):8-16.

4. Pappu R, Ramirez-Carrozzi V, Ota N, Ouyang W, Hu Y. The IL-17 family cytokines in immunity and disease. J Clin Immunol. 2010;30(2):185-95.

5. Wu D, Wu P, Huang Q, Liu Y, Ye J, Huang J. Interleukin-17: a promoter in colorectal cancer progression. Clin Dev Immunol. 2013;2013:436307.

6. Wu P, Wu D, Ni C, Ye J, Chen W, Hu G, et al. $\gamma \delta \mathrm{T} 17$ cells promote the accumulation and expansion of myeloid-derived suppressor cells in human colorectal cancer. Immunity. 2014;40(5):785-800.

7. Wang K, Kim MK, Di Caro G, Wong J, Shalapour S, Wan J, et al. Interleukin-17 receptor a signaling in transformed enterocytes promotes early colorectal tumorigenesis. Immunity. 2014;41(6):105263.

8. Wagsater D, Lofgren S, Hugander A, Dimberg J. Expression of interleukin-17 in human colorectal cancer. Anticancer Res. 2006;26(6B):4213-6.

9. Wang J, Xu K, Wu J, Luo C, Li Y, Wu X, et al. The changes of Th17 cells and the related cytokines in the progression of human colorectal cancers. BMC Cancer. 2012;12:418.

10. Grivennikov SI, Wang K, Mucida D, Stewart CA, Schnabl B, Jauch $\mathrm{D}$, et al. Adenoma-linked barrier defects and microbial products drive IL-23/IL-17-mediated tumour growth. Nature. 2012;491(7423):254-8. 
11. Galon J, Costes A, Sanchez-Cabo F, Kirilovsky A, Mlecnik B, Lagorce-Pages C, et al. Type, density, and location of immune cells within human colorectal tumors predict clinical outcome. Science. 2006;313(5795):1960-4.

12. Tosolini M, Kirilovsky A, Mlecnik B, Fredriksen T, Mauger S, Bindea $\mathrm{G}$, et al. Clinical impact of different classes of infiltrating T cytotoxic and helper cells (Th1, Th2, Treg, Th17) in patients with colorectal cancer. Cancer Res. 2011;71(4):1263-71.

13. Liu J, Duan Y, Cheng X, Chen X, Xie W, Long H, et al. IL-17 is associated with poor prognosis and promotes angiogenesis via stimulating VEGF production of cancer cells in colorectal carcinoma. Biochem Biophys Res Commun. 2011;407(2):348-54.

14. Chae WJ, Gibson TF, Zelterman D, Hao L, Henegariu O, Bothwell AL. Ablation of IL-17A abrogates progression of spontaneous intestinal tumorigenesis. Proc Natl Acad Sci USA. 2010;107(12): $5540-4$.

15. Wu S, Rhee KJ, Albesiano E, Rabizadeh S, Wu X, Yen HR, et al. A human colonic commensal promotes colon tumorigenesis via activation of T helper type $17 \mathrm{~T}$ cell responses. Nat Med. 2009;15(9): 1016-22.

16. Hayata K, Iwahashi M, Ojima T, Katsuda M, Iida T, Nakamori M, et al. Inhibition of IL-17A in tumor microenvironment augments cytotoxicity of tumor-infiltrating lymphocytes in tumor-bearing mice. PLoS One. 2013;8(1), e53131.

17. Kryczek I, Wei S, Szeliga W, Vatan L, Zou W. Endogenous IL-17 contributes to reduced tumor growth and metastasis. Blood. 2009; 114(2):357-9.

18. Bishehsari F, Mahdavinia M, Malekzadeh R, Verginelli F, Catalano $\mathrm{T}$, Sotoudeh M, et al. Patterns of K-ras mutation in colorectal carcinomas from Iran and Italy (a Gruppo Oncologico dell'Italia Meridionale study): influence of microsatellite instability status and country of origin. Ann Oncol. 2006;17 Suppl 7:vii91-6.

19. Al-Samadi A, Kouri VP, Salem A, Ainola M, Kaivosoja E, Barreto $\mathrm{G}$, et al. IL-17C and its receptor IL-17RA/IL-17RE identify human oral epithelial cell as an inflammatory cell in recurrent aphthous ulcer. J Oral Pathol Med. 2014;43(2):117-24.

20. Johnston A, Fritz Y, Dawes SM, Diaconu D, Al-Attar PM, Guzman AM, et al. Keratinocyte overexpression of IL-17C promotes psoriasiform skin inflammation. J Immunol. 2013;190(5):2252-62.

21. Fort MM, Cheung J, Yen D, Li J, Zurawski SM, Lo S, et al. IL-25 induces IL-4, IL-5, and IL-13 and Th2-associated pathologies in vivo. Immunity. 2001;15(6):985-95.

22. Kouri VP, Olkkonen J, Ainola M, Li TF, Bjorkman L, Konttinen YT, et al. Neutrophils produce interleukin-17B in rheumatoid synovial tissue. Rheumatology (Oxford). 2014;53(1):39-47.

23. Li H, Chen J, Huang A, Stinson J, Heldens S, Foster J, et al. Cloning and characterization of IL-17B and IL-17C, two new members of the IL-17 cytokine family. Proc Natl Acad Sci USA. 2000;97(2):773-8.
24. Yagi Y, Andoh A, Inatomi O, Tsujikawa T, Fujiyama Y. Inflammatory responses induced by interleukin-17 family members in human colonic subepithelial myofibroblasts. J Gastroenterol. 2007;42(9):746-53.

25. Huang $\mathrm{CK}$, Yang $\mathrm{CY}$, Jeng YM, Chen $\mathrm{CL}, \mathrm{Wu} \mathrm{HH}$, Chang $\mathrm{YC}$, et al. Autocrine/paracrine mechanism of interleukin-17B receptor promotes breast tumorigenesis through NF- $\mathrm{kB}$-mediated antiapoptotic pathway. Oncogene. 2014;33(23):2968-77.

26. Tazzyman S, Niaz H, Murdoch C. Neutrophil-mediated tumour angiogenesis: subversion of immune responses to promote tumour growth. Semin Cancer Biol. 2013;23(3):149-58.

27. Tecchio C, Cassatella MA. Neutrophil-derived cytokines involved in physiological and pathological angiogenesis. Chem Immunol Allergy. 2014;99:123-37.

28. Song X, Gao H, Lin Y, Yao Y, Zhu S, Wang J, et al. Alterations in the microbiota drive interleukin-17C production from intestinal epithelial cells to promote tumorigenesis. Immunity. 2014;40(1):140 52 .

29. Caruso R, Sarra M, Stolfi C, Rizzo A, Fina D, Fantini MC, et al. Interleukin-25 inhibits interleukin-12 production and Th1 celldriven inflammation in the gut. Gastroenterology. 2009;136(7): 2270-9.

30. McHenga SS, Wang D, Li C, Shan F, Lu C. Inhibitory effect of recombinant IL-25 on the development of dextran sulfate sodiuminduced experimental colitis in mice. Cell Mol Immunol. 2008;5(6):425-31.

31. Rizzo A, Monteleone I, Fina D, Stolfi C, Caruso R, Fantini MC, et al. Inhibition of colitis by IL-25 associates with induction of alternatively activated macrophages. Inflamm Bowel Dis. 2012;18(3):449-59.

32. McHenga SS, Wang D, Janneh FM, Feng Y, Zhang P, Li Z, et al. Differential dose effects of recombinant IL-25 on the development of dextran sulfate sodium-induced colitis. Inflamm Res. 2010;59(10):879-87.

33. Camelo A, Barlow JL, Drynan LF, Neill DR, Ballantyne SJ, Wong $\mathrm{SH}$, et al. Blocking IL-25 signalling protects against gut inflammation in a type- 2 model of colitis by suppressing nuocyte and NKT derived IL-13. J Gastroenterol. 2012;47(11):1198-211.

34. Tong Z, Yang XO, Yan H, Liu W, Niu X, Shi Y, et al. A protective role by interleukin- $17 \mathrm{~F}$ in colon tumorigenesis. PLoS One. 2012;7(4), e34959.

35. Xie Y, Sheng W, Xiang J, Ye Z, Yang J. Interleukin-17F suppresses hepatocarcinoma cell growth via inhibition of tumor angiogenesis. Cancer Invest. 2010;28(6):598-607.

36. Yamaguchi Y, Fujio K, Shoda H, Okamoto A, Tsuno NH, Takahashi K, et al. IL-17B and IL-17C are associated with TNFalpha production and contribute to the exacerbation of inflammatory arthritis. J Immunol. 2007;179(10):7128-36. 